\title{
Accuracy and predictive value of incarcerated adults' accounts of their self-harm histories: findings from an Australian prospective data linkage study
}

\author{
Rohan Borschmann PhD DClinPsych, Jesse T. Young MPH, Paul Moran MD, Matthew J. Spittal PhD, \\ Kathryn Snow MPH, Katherine Mok PhD, Stuart A. Kinner PhD
}

Abstract

Background: Self-harm is prevalent in prison populations and is a well-established risk factor for suicide. Researchers typically rely on self-report to measure self-harm, yet the accuracy and predictive value of self-report in prison populations is unclear. Using a large, representative sample of incarcerated men and women, we aimed to examine the level of agreement between self-reported self-harm history and historical medical records, and investigate the association between self-harm history and medically verified selfharm after release from prison.

Methods: During confidential interviews with 1315 adults conducted within 6 weeks of expected release from 1 of 7 prisons in Queensland, Australia, participants were asked about the occurrence of lifetime self-harm. Responses were compared with prison medical records and linked both retrospectively and prospectively with ambulance, emergency department and hospital records to identify instances of medically verified self-harm. Follow-up interviews roughly 1, 3 and 6 months after release covered the same domains assessed in the baseline interview as well as self-reported criminal activity and contact with health care, social and criminal justice services since release.

Results: Agreement between self-reported and medically verified history of self-harm was poor, with 64 (37.6\%) of 170 participants with a history of medically verified self-harm disclosing a history of self-harm at baseline. Participants with a medically verified history of self-harm were more likely than other participants to self-harm during the follow-up period. Compared to the unconfirmed-negative group, the true-positive (adjusted hazard ratio [HR] 6.2 [95\% confidence interval (Cl) 3.3-10.4]), false-negative (adjusted HR 4.0 [95\% Cl 2.2-6.7]) and unconfirmed-positive (adjusted HR 2.2 [95\% Cl 1.2-3.9]) groups were at increased risk for self-harm after release from prison.

Interpretation: Self-reported history of self-harm should not be considered a sensitive indicator of prior self-harm or of future selfharm risk in incarcerated adults. To identify those who should be targeted for preventive strategies, triangulation of data from multiple verifiable sources should be performed whenever possible.

1 he incidence of self-harm is markedly higher among incarcerated ${ }^{1,2}$ and formerly incarcerated ${ }^{3,4}$ adults than in the general population. Although one of the strongest predictors of future self-harm is a history of self-harm, ${ }^{5,6}$ repetition of self-harm is often difficult to predict with any degree of accuracy. ${ }^{7,8}$ In many prison settings internationally, screening for self-harm history at prison reception is conducted exclusively by self-report. ${ }^{9}$ One purpose of screening is to identify people who may benefit from access to mental health services during and after incarceration. However, the validity of self-reported self-harm in this population remains unknown. Accurate identification of previous self-harm events could help to minimize the risk to these people during the transition from custody, a period characterized by elevated rates of suicide and all-cause mor- tality. ${ }^{10-13}$ The few studies of community populations have shown poor agreement between self-reported and medically verified self-harm, ${ }^{14}$ with participants providing conflicting accounts of their self-harm histories at different times. ${ }^{15,16}$

Self-harm is highly stigmatized, ${ }^{17}$ and this may lead to underreporting of the behaviour. ${ }^{18}$ Although most self-harm is not associated with help-seeking behaviour, ${ }^{19}$ medically severe

Competing interests: None declared.

This article has been peer reviewed.

Correspondence to: Rohan Borschmann, rohan.borschmann@mcri. edu.au

CMAJ Open 2017. DOI:10.9778/cmajo.20170058 
events typically result in contact with health care services such as ambulance, emergency department or hospital. ${ }^{20}$ Further complicating disclosure of self-harm in correctional settings is the prospect that doing so may result in intrusive observations or monitoring. Self-reported self-harm may therefore fail to identify a proportion of people at risk for subsequent (and medically more severe) self-harm and associated mental health problems. Underreporting may be particularly pronounced among those already marginalized, such as Indigenous people,$^{21}$ people with a mental illness ${ }^{6}$ and those who identify as lesbian, gay, bisexual or transgender. ${ }^{22,23}$ In Canada, historical underascertainment of self-harm events combined with a sharp increase in such events in prisons ${ }^{24,25}$ has led to recent changes in reporting practices in the Correctional Service of Canada, such as allowing staff to report 1 incident under multiple incident categories. ${ }^{26,27}$

Self-harm in incarcerated populations is prevalent in many countries including Canada, ${ }^{28}$ England and Wales, ${ }^{1}$ the United States, ${ }^{29}$ Australia, ${ }^{30}$ France $^{31}$ and Greece. ${ }^{32}$ There are, however, few studies examining self-harm after release from incarceration, which makes direct comparison between regions almost impossible. ${ }^{3,4}$ In Australia, there is no federal correctional system, as each state or territory system operates independently. In the State of Queensland, adults are asked on reception whether they have ever selfharmed; this is combined with records from previous incarcerations and, on some occasions, information gathered informally from police, mental health records and family members. However, this is provided on an ad hoc basis only, with no system in place for routinely obtaining such collateral information.

In a large cohort of incarcerated adults in Australia, we aimed to determine the level of agreement between selfreported history of self-harm and medically verified history of self-harm. We also aimed to examine the extent to which selfreported history of self-harm, a medically verified history of self-harm and a combination of the 2 predicted the occurrence of future medically verified self-harm following release from prison.

\section{Methods}

\section{Participants}

The participants were 1325 adult prisoners recruited to the Passports study ${ }^{33,34}$ - a randomized controlled trial of a service brokerage intervention, but here analyzed as a cohort study - within 6 weeks of expected release from 1 of 7 prisons in Queensland. Trained researchers, independent of Queensland Corrective Services, obtained a list of all potentially eligible participants for each prison. Prisoners identified as potentially eligible were approached by researchers and invited to participate in the study; this included screening for eligibility, explaining the project in plain language and obtaining informed, written consent. Researchers recorded the outcome of each approach (not eligible, eligible [participated or declined] or unknown [missed or unavailable]) to permit calculation of a recruitment factor.

\section{Inclusion and exclusion criteria}

To maximize generalizability, eligibility criteria were as inclusive as possible. Inclusion criteria were 1) sentenced adult prisoner expecting to be released (full-time or on parole) from 1 of the 7 recruitment prisons within the next 6 weeks, 2) judged safe to be approached and 3) able to provide informed, written consent. Exclusion criteria were 1) on remand (owing to uncertainty around release) and 2) having previously participated in the trial (necessitated by the high rate of recidivism in the population). We intentionally oversampled women to increase the sample size for sex-stratified analyses. To permit identification of and adjustment for sampling bias, we obtained demographic and offending information from Queensland Corrective Services for all sentenced prisoners released from custody in Queensland during the 2 years of recruitment $(n=10931)$.

\section{Data collection}

\section{Survey data}

Baseline data were collected during confidential, structured, face-to-face interviews that covered demographic characteristics, physical and mental health, self-harm history, substance use before and during incarceration, and other health-related risk behaviours. The interviews were conducted between August 2008 and July 2010. Interviewers were experienced with interviewing vulnerable populations about sensitive topics, were extensively trained (including by a psychologist) and were independent of both corrections and the prison health care service. Substantial reporting of stigmatized behaviours, including behaviours that could have implications for sentencing, ${ }^{35}$ indicated a high degree of rapport between participants and interviewers. Participants were advised that they were not required to answer any questions that they did not wish to, but that all responses would be kept strictly confidential. Participants in the intervention group received a personalized booklet summarizing their health status and medication needs, and identifying appropriate community health care services; trained workers made weekly telephone contact in the first 4 weeks after release to identify emergent health needs and promote contact with health care services. Participants in the control arm received usual care. Follow-up interviews, completed by December 2010, occurred by telephone roughly 1, 3 and 6 months after release. Although considerably briefer, they covered the same domains assessed in the baseline interview as well as selfreported criminal activity and contact with health care, social and criminal justice services since release. The Passports study is described in greater detail elsewhere. ${ }^{33,34}$

\section{Administrative and clinical data}

We obtained emergency department records from June 1, 2002, to July 31, 2012, ambulance records from Jan. 1, 2007, to Jan. 1, 2014, and hospital records from July 1, 1999, to July 31, 2012. Baseline interview data were linked probabilistically with clerical review by the Queensland Health Data Linkage Unit using full name and any known aliases, sex, date of birth and postcode of last residence. Validation studies and quality 
assurance checks from Australian jurisdictions with comparable linkage processes have shown that this method produces false-positive and false-negative rates less than $0.1 \% .^{20,36-39}$ The inclusion of all known aliases as a linkage identifier (considered the gold standard for research involving vulnerable populations) has been shown to significantly increase the sensitivity of matching while maintaining the same level of specificity. ${ }^{40}$ Linkage with correctional records and prison medical records was deterministic, based on a unique prisoner identification number. Prison admission and release dates were identified from Sept. 1, 2008, to Dec. 31, 2013 from Queensland Corrective Services records. Queensland Corrective Services data also contained a dichotomous variable indicating whether each participant had been identified by any staff member at any time as being at risk for self-harm/suicide. Prison medical records for participants were coded by the research team using the International Classification for Primary Care Version $2 .^{41}$ In addition, coders recorded relevant contextual information, including regarding instances of self-harm, in a free-text field.

\section{Measures}

\section{Self-harm}

Self-reported: During the baseline interview (and after rapport had been established), participants were asked "Have you ever attempted suicide?" Participants were then asked "Apart from suicide attempts, have you ever deliberately harmed or injured yourself?" Participants who responded in the affirmative were asked a follow-up question: "If yes, how many times have you deliberately harmed or injured yourself?" Participants who responded in the affirmative to either of these stem questions (irrespective of the severity of harm) were recorded as having a self-reported history of self-harm.

Medically verified: We searched all emergency department and hospital records for International Classification of Diseases diagnosis codes for self-harm (X60-X84) and searched the International Classification for Primary Care Version 2 coding of the free-text field of all prison medical records for all participants to identify self-harm events. In addition, freetext fields in all ambulance and emergency department records and all free-text notes made by the coding staff who abstracted the prison medical records were screened by a member of the study team (K.M.) to increase case ascertainment. K.M. was blinded to participants' self-harm status during the screening process. Free-text data were coded with the use of a system adapted from a large epidemiological study of self-harm. ${ }^{42}$ All emergency department, ambulance and prison health records for all participants were coded as either 0 (did not involve self-harm) or 1 (involved self-harm), with the latter category comprising any contact with health care services resulting from behaviours fitting into any 1 of 5 categories: 1) cutting/burning, 2) self-poisoning, 3) self-battering, 4) nonrecreational risk-taking or 5) other self-harm. Any contact with health care services before the baseline interview that was deemed to have resulted from self-harm was coded as a medically verified self-harm event. Because participants may have self-harmed in the past without self-disclosing this at baseline or without presenting to health care services as a result, neither a failure to appear in the health records nor a failure to self-report a history of self-harm was taken as a definitive indication of absence of historical self-harm. Time at risk commenced at the initial prison release date and was censored on the last day of the follow-up period (Jan. 1, 2014), return to custody or death, whichever came first.

\section{Categorization of self-harm history}

Based on the combination of their baseline interview responses and retrospectively linked health records, participants were categorized into 1 of 4 mutually exclusive groups:

- Unconfirmed-negative case: participant did not disclose self-harm at baseline interview and had no medically verified self-harm events before baseline

- False-negative case: participant did not disclose self-harm at baseline interview but had 1 or more medically verified self-harm events before baseline

- Unconfirmed-positive case: participant disclosed self-harm at baseline interview but had no medically verified selfharm events before baseline

- True-positive case: participant disclosed self-harm at baseline interview and had 1 or more medically verified selfharm events before baseline.

\section{Statistical analysis}

We calculated descriptive statistics for all measures. We compared differences in participants' baseline characteristics across the self-harm categories using $\chi^{2}$ tests. To estimate the agreement between self-reported and medically verified self-harm, we calculated prevalence-adjusted and bias-adjusted $\kappa$ statistics, as recommended when comparing administrative data as a reference standard and for low prevalence outcomes. ${ }^{43,44}$ In addition, we calculated the average positive and negative agreement, asserted as best practice when assessing agreement. ${ }^{45}$ Crude incidence rates and $95 \%$ confidence intervals (CIs) for medically verified self-harm after release from custody were calculated overall and separately for each self-harm category. We estimated the conditional probability of survival to study end without evidence of medically verified self-harm after release from custody by fitting a Kaplan-Meier plot. We estimated the risk of medically verified self-harm after release from custody for each self-harm agreement group by fitting univariate and multivariate Cox proportional hazards models. ${ }^{46}$ We calculated standard errors and bias-corrected 95\% CIs of the Cox model parameters using the bootstrap method $(n=$ 1000 repetitions). ${ }^{47}$ The multivariate model was adjusted for sex, age, Indigenous status, relationship status, years of education, sexual identity, Queensland Corrective Services self-harm risk flag, prior suicide attempt, lifetime history of mental disorder and whether the most serious offence was violent (including sex offences) or nonviolent (including offences against property, drug trafficking, driving offences and forgery/fraud). The inclusion of violent offences in the model was based on the known association between violent offending and increased risk of both self-harm ${ }^{48,49}$ and suicide..$^{50,51}$ This coding was per- 
formed manually based on the Australian Standard Offence Classification (Queensland Extension). ${ }^{52}$ In light of the reported sex differences in self-harm prevalence, ${ }^{6}$ we examined the possibility of effect modification in the adjusted model by fitting interaction terms between self-harm category at baseline and demographic variables (sex, age and Indigenous status). The Cox model was censored at date of death, the first medically verified self-harm event or the last day of the followup period, whichever occurred first. We conducted all analyses using STATA, version 14.2.

\section{Ethics approval}

Ethics approval was granted by The University of Queensland's Behavioural and Social Sciences Ethical Review Committee, the Queensland Health Human Research Ethics Committee and the Queensland Corrective Services Research Committee.

\section{Results}

We conducted all analyses using the data for the 1315 participants $(99.2 \%)$ for whom linked health and correctional records were obtained (Figure 1). Table 1 describes the baseline characteristics of the participants. Of the 1315 participants, 1037 (78.9\%) were men, 336 (25.6\%) were Indigenous, and just over half (681 [51.8\%]) were aged $25-39$ years.

\section{Self-harm}

\section{Historical}

Of the 1315 participants, 186 (14.1\%) disclosed a history of self-harm at the baseline interview, and 170 (12.9\%) (119 men and 51 women) had 1 or more medically verified self-harm events recorded before their baseline interview. Of the 170 ,

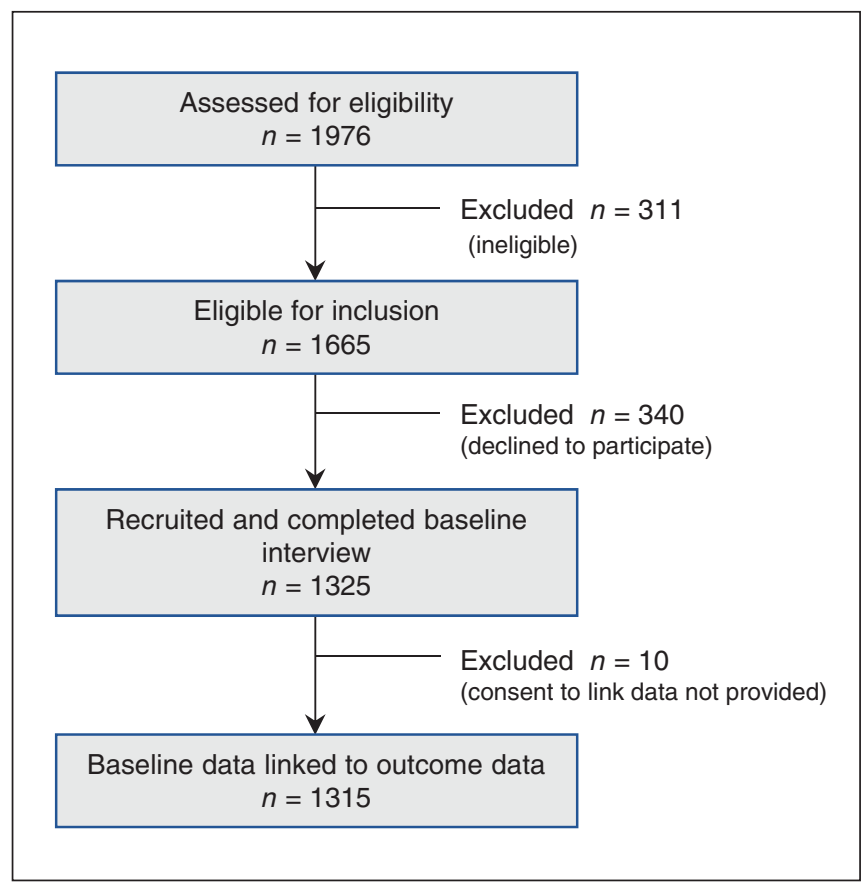

Figure 1: Participant flow through the Passports study.

$64(37.6 \%)$ disclosed a history of self-harm and were categorized as true-positive cases; the remaining 106 (62.4\%) were categorized as false-negative cases. Of the 1145 participants who had no medically verified record of self-harm at baseline, $122(10.6 \%)$ reported a history of self-harm and were categorized as unconfirmed-positive cases; the remaining 1023 $(89.3 \%)$ were categorized as unconfirmed-negative cases. Table 2 displays the relation between self-reported and medically verified self-harm. Most (106 [62.4\%]) of the 170 participants with a medically verified history of self-harm did not disclose this at baseline. Disclosure of prior self-harm was uncommon regardless of participant sex, Indigenous status, education or history of mental disorder. Appendix 1, Supplementary Table 1 (available at www.cmajopen.ca/content/5/3/ E694/suppl/DC1) displays the demographic and criminogenic characteristics of the cohort at baseline, according to selfharm category.

Overall, prevalence-adjusted and bias-adjusted agreement between self-reported and medically verified self-harm was estimated at 0.65 (95\% CI 0.61-0.69). Average positive agreement was 0.36 (95\% CI $0.30-0.42)$, and average negative agreement was 0.90 (95\% CI 0.89-0.91). Agreement greater than 0.8 is considered "almost perfect," and agreement of $0.21-0.4$ is considered "fair." 53

Table 1: Baseline characteristics of participants

\begin{tabular}{|c|c|}
\hline Characteristic & $\begin{array}{c}\text { No. (\%) of } \\
\text { participants } \\
n=1315\end{array}$ \\
\hline Male sex & $1037(78.9)$ \\
\hline \multicolumn{2}{|l|}{ Age at release, yr } \\
\hline $18-24$ & $336(25.6)$ \\
\hline 25-39 & $681(51.8)$ \\
\hline$\geq 40$ & $298(22.7)$ \\
\hline Indigenous & $336(25.6)$ \\
\hline \multicolumn{2}{|l|}{ Relationship status } \\
\hline Married or de facto relationship & $452(34.4)$ \\
\hline Other & $863(65.6)$ \\
\hline$<10 \mathrm{yr}$ of education & $574(43.7)$ \\
\hline \multicolumn{2}{|l|}{ Sexuality } \\
\hline Heterosexual & $1234(93.9)$ \\
\hline Lesbian/gay/bisexual//transgender & $80(6.1)$ \\
\hline Missing & $1(0.1)$ \\
\hline $\begin{array}{l}\text { Queensland Corrective Services history } \\
\text { of self-harm flag }\end{array}$ & $292(22.4)$ \\
\hline Previous suicide attempt* & $280(21.3)$ \\
\hline Lifetime history of any mental disorder* & $572(43.5)$ \\
\hline $\begin{array}{l}\text { Previous hospital admission for mental } \\
\text { disorder }\end{array}$ & $125(9.6)$ \\
\hline Violent offence (index incarceration) & $684(52.7)$ \\
\hline
\end{tabular}




\section{After release from prison}

A total of 123 participants (9.4\%) had 1 or more medically verified self-harm events recorded after release from custody during a median of 854 (interquartile range 219-1560) days of follow-up. Table 3 displays the proportion of participants with 1 or more medically verified self-harm events across demographic groups. The crude incidence rates per 1000 personyears for the 4 self-harm categories were as follows: truepositive cases 431.8 (95\% CI 326.3-571.3), false-negative cases 311.5 (95\% CI 238.0-407.8), unconfirmed-positive cases 105.1 (95\% CI 71.6-154.4) and unconfirmed-negative cases 35.6 (95\% CI 28.7-44.3). The full multivariate model is shown in Appendix 1, Supplementary Table 2.

About 4 in 10 (27 [42.2\%]) participants in the true-positive group had 1 or more medically verified self-harm events during the follow-up period, almost twice as many as in any of the other self-harm groups. The proportion of participants with medically verified self-harm events during follow-up was higher among women than men, and among Indigenous than non-Indigenous participants. However, sex, age and Indigenous status did not significantly modify the relation between self-harm category at baseline and medically verified selfharm after release from custody $(p=1.0, p=0.8$ and $p=0.6$, respectively). Figure 2 shows the survival function for medically verified self-harm events after release from custody, according to baseline self-harm category.

\section{Interpretation}

In this representative cohort of incarcerated adults in Australia, agreement regarding history of self-harm events between selfreport and health care service records was poor: just $38 \%$ of participants with a medically verified history of self-harm disclosed this history during the baseline interview. After adjustment for covariate effects, participants with both a medically verified and a self-reported history of self-harm were 6 times more likely to have self-harmed during the follow-up period than participants in the reference category (unconfirmednegative group). Those who had a medically verified history of self-harm but did not disclose this at baseline were 4 times more likely to have self-harmed during the follow-up period than participants in the reference category. Finally, participants who disclosed a history of self-harm but had no medically verified self-harm events in their records were twice as likely to have a medically verified self-harm event during the follow-up period than participants in the reference category.

There are several potential explanations for the observed low prevalence of self-reported self-harm at baseline. First, selfharm is still strongly stigmatized, ${ }^{17}$ and social desirability bias may have contributed to failure to disclose prior self-harm. Although the research team was independent of corrective services and interviews were carried out in private, it is possible that some participants with a history of self-harm may have

\begin{tabular}{|lccc|}
\hline \multicolumn{4}{|l|}{$\begin{array}{l}\text { Table 2: Two-by-two table showing proportions of participants assigned to the } \\
\mathbf{4} \text { mutually exclusive self-harm categories }\end{array}$} \\
\hline \multicolumn{4}{|c|}{ Medically verified self-harm; no. (\%) of participants } \\
$n=1315$
\end{tabular}

\begin{tabular}{|c|c|c|c|c|}
\hline \multirow[b]{2}{*}{ Baseline self-harm category } & \multicolumn{2}{|c|}{$\begin{array}{l}\text { No. of events recorded after } \\
\text { release; no. (\%) of participants }\end{array}$} & \multirow[b]{2}{*}{$\mathrm{HR}(95 \% \mathrm{Cl})$} & \multirow{2}{*}{$\begin{array}{l}\text { Adjusted HR } \\
(95 \% \mathrm{Cl})^{*}\end{array}$} \\
\hline & 0 & $\geq 1$ & & \\
\hline True positive $(n=64)$ & $35(54.7)$ & $27(42.2)$ & $10.6(6.5-17.2)$ & $6.2(3.5-10.8)$ \\
\hline False negative $(n=106)$ & $82(77.4)$ & $24(22.6)$ & $5.8(3.5-9.5)$ & $4.0(2.3-7.0)$ \\
\hline Unconfirmed positive $(n=122)$ & $101(82.8)$ & $19(15.6)$ & $3.4(2.0-5.9)$ & $2.2(1.2-4.1)$ \\
\hline Unconfirmed negative $(n=1023)$ & $965(94.3)$ & $53(5.2)$ & Reference & Reference \\
\hline \multicolumn{5}{|c|}{$\begin{array}{l}\text { Note: } \mathrm{Cl}=\text { confidence interval, } \mathrm{HR}=\text { hazard ratio. } \\
\text { *Adjusted for sex, age, Indigenous status, relationship status, years of education, sexual identity, Queensland Corrective Services } \\
\text { self-harm risk flag, prior suicide attempt, lifetime history of mental disorder and violent offence. }\end{array}$} \\
\hline
\end{tabular}




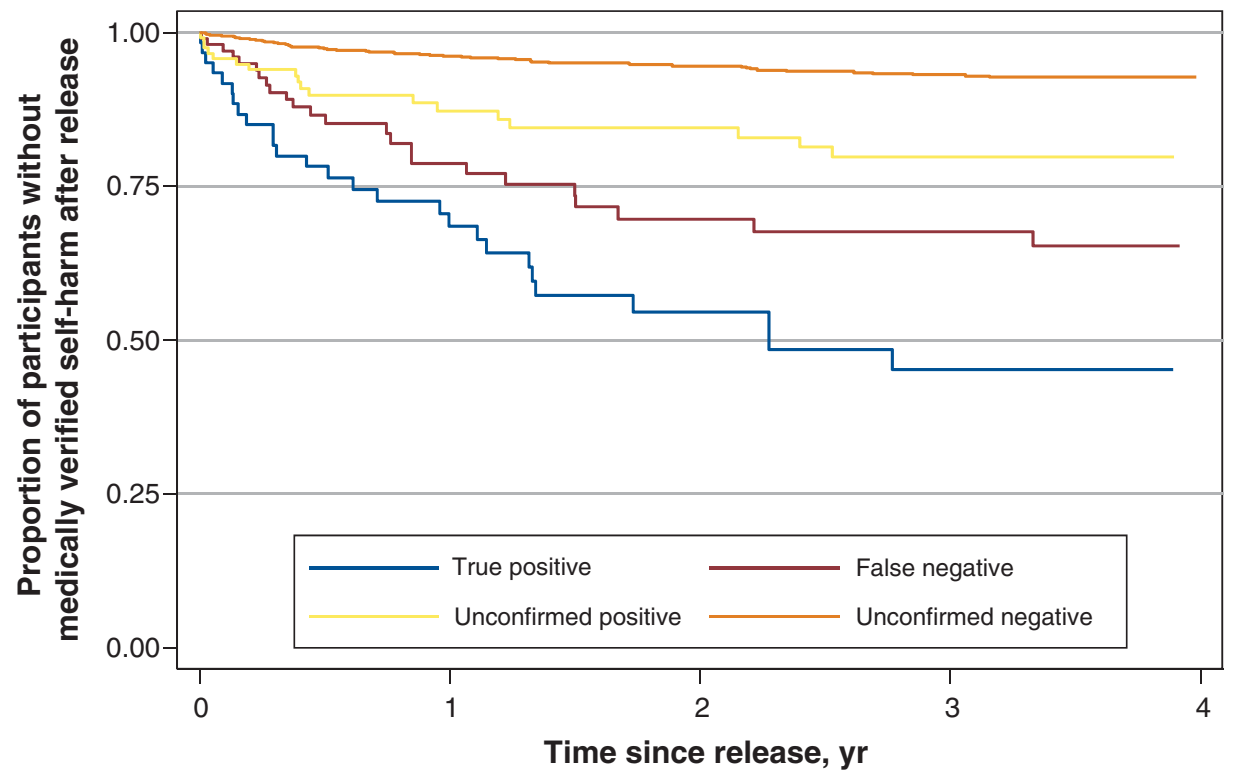

No. at risk

$\begin{array}{lr}\text { True positive } & 61 \\ \text { False negative } & 106 \\ \text { Unconfirmed positive } & 121 \\ \text { Unconfirmed negative } & 1021\end{array}$

33

47

67

679

19
35
54
543

19

35

543

$\begin{array}{rr}12 & 8 \\ 31 & 21 \\ 48 & 35 \\ 481 & 346\end{array}$

Figure 2: Kaplan-Meier curve of medically verified self-harm events in the first 4 years following release from prison according to self-harm category.

incorrectly believed that a disclosure of self-harm would have an adverse impact on their current incarceration and/or delay their impending release. Second, participants may have had different perceptions about what constitutes self-harm or may not have been able to recall previous events. Although we might have been able to increase ascertainment through more detailed questioning, to the extent that our approach to questioning replicates intake screening in prison settings, this limitation is in fact an accurate assessment of routine practice.

In addition to the high proportion of people with a medically verified history of self-harm who did not disclose this at baseline, we also observed the inverse phenomenon: 1 in 9 participants (11\%) reported a history of self-harm at baseline that did not appear in their medical records (unconfirmed-positive group). The most likely explanation for this finding is that most self-harm does not lead to formal help-seeking behaviour, ${ }^{54}$ which makes it more difficult than other clinical phenomena to measure accurately through medical records..$^{55}$ Our finding that people in the unconfirmed-positive group were at increased risk for self-harm following release from incarceration suggests that their self-reported histories were accurate. As such, although self-reported self-harm and medically verified self-harm had low agreement in our sample, there is merit in inquiring about prior self-harm, as it can identify a subgroup of people who may not yet have come to the attention of mental health services but who may nevertheless be at increased risk for further self-harm. Other potential explanations for the high proportion of participants in this group include failure by clinicians to document the detection of self-harm in some cases, which would have led to underascertainment of selfharm in medical records, and reporting of incidents that participants considered self-harm but that would not have been identified as such by health care professionals (e.g., hair pulling, lip biting or putting oneself at risk from others).

We were unable to identify other studies examining the association between incarcerated adults' self-reported and medically verified self-harm history, and medically verified self-harm after release from prison.

Accurately ascertaining self-harm events is vital for determining the morbidity and mortality associated with this set of behaviours. ${ }^{56,57}$ Our findings indicate that relying solely on a self-reported history of self-harm in incarcerated adults substantially underascertains actual history, which suggests that many vulnerable people will be missed if this is the sole method for screening in prison settings.

One clear implication of our findings is that a more comprehensive method of identifying people at risk for self-harm is to triangulate data from multiple sources including self-report, 
clinician/observer ratings (e.g., from prison medical records) and community medical records. ${ }^{58}$ Consistent with this, our findings suggest that incarcerated adults who disclose a history of self-harm and have a medically verified record of self-harm are at considerably increased risk for further self-harm after release from custody (almost 1 in 2 in our study) and may require additional community-based support to prevent these events. Identifying these people to provide such support is likely to require access to historical medical records. Incarcerated people who disclose a history of self-harm, even without a medically verified history, are at moderately increased risk for engaging in self-harm after release from prison (about 1 in 5 in our study) and are also likely to require targeted support and preventive strategies.

\section{Strength and limitations}

This study has important strengths. It used 4 separate, unique data sources to ascertain episodes of medically verified selfharm. In addition, we linked highly accurate administrative data with rich baseline interview data, optimizing the ascertainment of key demographic, criminogenic and exposure data. ${ }^{59}$

Most limitations of our study would have resulted in underascertainment of self-harm events and thus rendered our estimates conservative. First, although we were able to verify the true-positive and false-negative categories objectively, the same cannot be said for the unconfirmed-positive and unconfirmed-negative categories. Because prior self-harm events that did not result in medical treatment (and were not disclosed) would not have been recorded in any of the 4 selfharm categories, our estimates of the proportion of participants with a history of self-harm would be correspondingly conservative. Second, we did not have complete historical information from medical records for all participants, and, as such, it is likely that we underascertained self-harm events. Third, neither a failure to appear in the medical records nor a failure to self-report a history of self-harm was taken as an indication of absence of prior self-harm; consequently, we could not calculate the specificity of self-reported self-harm. Fourth, we did not have a sufficient number of events to examine the possibility of a dose-response relation between episodes of reincarceration and patterns of self-harm, similar to the pattern that exists between episodes of incarceration and death. ${ }^{60}$ Future studies should treat reincarceration as a time-varying covariate instead of censoring at reincarceration. Fifth, although we used Jan. 1, 2014 as our censoring date, the ambulance and hospital records ended on July 31, 2012. This would have resulted in underascertainment of medically verified self-harm events and likely attenuated the observed associations between self-harm history and medically verified selfharm. Finally, presentations to health care services outside Queensland would not have appeared in the data to which we had access. However, additional linked data relating to study participants indicated that less than $5 \%$ of the sample accessed health care services solely outside Queensland (mirroring recent research ${ }^{61}$ ), and, as such, the number of health presentations due to self-harm outside Queensland is likely to have been small. ${ }^{62}$

\section{Conclusion}

Self-report appears to be an insensitive method for documenting prior self-harm in incarcerated adults. However, selfreport is the sole method for intake screening in many prison settings and is subsequently relied on heavily to identify those at risk. The findings of the current study support the use of linked medical records to supplement self-reported indicators of self-harm wherever feasible (with appropriate participant consent), particularly when ascertainment of such events informs allocation of scarce mental health resources in and after release from prison. It is likely that, as with the measurement of violent behaviour, the most accurate way of ascertaining self-harm events would involve triangulating data from multiple sources.

\section{References}

1. Hawton K, Linsell L, Adeniji T, et al. Self-harm in prisons in England and Wales: an epidemiological study of prevalence, risk factors, clustering, and subsequent suicide. Lancet 2014;383:1147-54.

2. Lohner J, Konrad N. Deliberate self-harm and suicide attempt in custody: distinguishing features in male inmates' self-injurious behavior. Int 7 Law Psychiatry 2006;29:370-85.

3. Borschmann R, Thomas E, Moran P, et al. Self-harm following release from prison: a prospective data linkage study. Aust N Z J Psychiatry 2017;51:250-9.

4. Borschmann R, Young JT, Moran P, et al. Ambulance attendances resulting from self-harm after release from prison: a prospective data linkage study. Soc Psychiatry Psychiatr Epidemiol 2017 Apr. 7 [Epub ahead of print]. doi:10.1007/ s00127-017-1383-z.

5. Glenn CR, Klonsky ED. Prospective prediction of nonsuicidal self-injury: a 1-year longitudinal study in young adults. Behav Ther 2011;42:751-62.

6. Skegg K. Self-harm. Lancet 2005;366:1471-83.

7. Quinlivan L, Cooper J, Meehan D, et al. Predictive accuracy of risk scales following self-harm: multicentre, prospective cohort study. $\mathrm{Br} \mathcal{F}$ Psychiatry 2017;210:429-36.

8. Bilén K, Ponzer S, Ottosson C, et al. Deliberate self-harm patients in the emergency department: Who will repeat and who will not? Validation and development of clinical decision rules. Emerg Med f 2013;30:650-6.

9. Perry AE, Marandos R, Coulton S, et al. Screening tools assessing risk of suicide and self-harm in adult offenders: a systematic review. Int 7 Offender Ther Comp Criminol 2010;54:803-28.

10. Binswanger IA, Blatchford PJ, Mueller SR, et al. Mortality after prison release: opioid overdose and other causes of death, risk factors, and time trends from 1999 to 2009. Ann Intern Med 2013;159:592-600.

11. Kariminia A, Law MG, Butler TG, et al. Suicide risk among recently released prisoners in New South Wales, Australia. Med 7 Aust 2007;187:387-90.

12. Pratt D, Piper M, Appleby L, et al. Suicide in recently released prisoners: a population-based cohort study. Lancet 2006;368:119-23.

13. Spittal MJ, Forsyth S, Pirkis J, et al. Suicide in adults released from prison in Queensland, Australia: a cohort study. 7 Epidemiol Community Health 2014;68: 993-8.

14. Mitchell AJ, Hussain S, Leaver J, et al. Is there a difference between hospitalverified and self-reported self-harm? Implications for repetition. Gen Hosp Psychiatry 2016;43:12-6.

15. Mars B, Cornish R, Heron J, et al. Using data linkage to investigate inconsistent reporting of self-harm and questionnaire non-response. Arch Suicide Res 2016;20:113-41.

16. Velting DM, Rathus JH, Asnis GM. Asking adolescents to explain discrepancies in self-reported suicidality. Suicide Life Threat Behav 1998;28:187-96.

17. Mackay N, Barrowclough C. Accident and emergency staff's perceptions of deliberate self-harm: attributions, emotions and willingness to help. $\mathrm{Br} F \mathrm{Clin}$ Psychol 2005;44:255-67.

18. Rowe SL, French RS, Henderson $\mathrm{C}$, et al. Help-seeking behaviour and adolescent self-harm: a systematic review. Aust N Z F Psychiatry 2014;48:1083-95.

19. Nada-Raja S, Morrison D, Skegg K. A population-based study of help-seeking for self-harm in young adults. Aust NZ 7 Psychiatry 2003;37:600-5.

20. Lloyd B, Gao CX, Heilbronn C, et al. Self-harm and mental health-related ambulance attendances in Australia: 2013 data. Fitzroy (Australia): Turning Point; 2015.

21. Farrelly T, Francis K. Definitions of suicide and self-harm behavior in an Australian aboriginal community. Suicide Life Threat Behav 2009;39:182-9.

22. Deliberto TL, Nock MK. An exploratory study of correlates, onset, and offset of non-suicidal self-injury. Arch Suicide Res 2008;12:219-31.

23. Liu RT, Mustanski B. Suicidal ideation and self-harm in lesbian, gay, bisexual, and transgender youth. Am F Prev Med 2012;42:221-8. 
24. Risky business: an investigation into the treatment and management of chronic selfinjury among federally sentenced women. Ottawa: Office of the Correctional Investigator; 2013

25. Gordon A. Self-injury incidents in CSC institutions over a thirty-month period. Research report R-233. Ottawa: Correctional Service of Canada; 2010.

26. Martin MS, Dorken SK, Colman I, et al. The incidence and prediction of self-injury among sentenced prisoners. Can 7 Psychiatry 2014;59:259-67.

27. Management of inmate self-injurious and suicidal behaviour. Ottawa: Correctional Service Canada; 2013. Available: www.csc-scc.gc.ca/acts-and -regulations/843-cd-eng.shtml\#s2a (accessed 2016 Nov. 19).

28. Wichmann C, Serin R, Abracen J. Women offenders who engage in self-barm: a comparative investigation. Ottawa: Research Branch, Correctional Service Canada; 2002

29. Kaba F, Lewis A, Glowa-Kollisch S, et al. Solitary confinement and risk of self-harm among jail inmates. Am 7 Public Health 2014:104:442-7.

30. Dear GE, Thomson DM, Howells K, et al. Self-harm in Western Australian prisons: differences between prisoners who have self-harmed and those who have not. Aust N Z 7 Criminol 2001;34:277-92.

31. Godet-Mardirossian H, Jehel L, Falissard B. Suicidality in male prisoners: influence of childhood adversity mediated by dimensions of personality. 7 Forensic Sci 2011;56:942-9.

32. Fotiadou M, Livaditis M, Manou I, et al. Prevalence of mental disorders and deliberate self-harm in Greek male prisoners. Int 7 Law Psychiatry 2006;29:68-73.

33. Kinner SA, Lennox N, Williams GM, et al. Randomised controlled trial of a service brokerage intervention for ex-prisoners in Australia. Contemp Clin Trials 2013;36:198-206.

34. Kinner SA, van Dooren K, Boyle FM, et al. Development of an intervention to increase health service utilisation in ex-prisoners. Health fustice 2014;2:4

35. Kinner SA, Jenkinson R, Gouillou M, et al. High-risk drug-use practices among a large sample of Australian prisoners. Drug Alcohol Depend 2012;126:156-60.

36. Preen DB, Holman CAJ, Lawrence DM, et al. Hospital chart review provided more accurate comorbidity information than data from a general practitioner survey or an administrative database. 7 Clin Epidemiol 2004;57:1295-304.

37. Lawrence G, Dinh I, Taylor L. The Centre for Health Record Linkage: a new resource for health services research and evaluation. Health Inf Manage 7 2008;37:60-2.

38. Boyd JH, Ferrante AM, O'Keefe CM, et al. Data linkage infrastructure for cross-jurisdictional health-related research in Australia. BMC Health Serv Res 2012;12:480

39. Brameld KJ, Thomas MAB, Holman CAJ, et al. Validation of linked administrative data on end-stage renal failure: application of record linkage to a 'clinical base population.' Aust N Z7 Public Health 1999;23:464-7.

40. Larney S, Burns L. Evaluating health outcomes of criminal justice populations using record linkage: the importance of aliases. Eval Rev 2011;35:118-28

41. O'Halloran J, Miller GC, Britt H. Defining chronic conditions for primary care with ICPC-2. Fam Pract 2004;21:381-6.

42. Moran P, Coffey C, Romaniuk H, et al. The natural history of self-harm during adolescence and young adulthood: population-based cohort study. Lancet 2012;379:236-43

43. Looney SW, Hagan JL. Statistical methods for assessing biomarkers and analyzing biomarker data. In: Rao CR, Miller J, Rao DC, editors. Epidemiology and medical statistics. vol. 27 of Handbook of statistics series. Amsterdam: Elsevier; 2008.

44. Chen G, Faris P, Hemmelgarn B, et al. Measuring agreement of administrative data with chart data using prevalence unadjusted and adjusted kappa. BMC Med Res Methodol 2009;9:5

45. Cicchetti DV, Feinstein AR. High agreement but low kappa: II. Resolving the paradoxes. 7 Clin Epidemiol 1990;43:551-8.

46. Cox DR. Regression models and life-tables. 7 R Stat Soc Series B Stat Methodol 1972;34:187-220.

47. Efron B, Tibshirani R. Bootstrap methods for standard errors, confidence intervals, and other measures of statistical accuracy. Stat Sci 1986;1:54-75.

48. Jordan JT, Samuelson KW. Predicting suicide intent: the roles of experiencing or committing violent acts. Suicide Life Threat Behav 2016;46:293-300.

49. Webb RT, Antonsen S, Carr MJ, et al. Self-harm and violent criminality among young people who experienced trauma-related hospital admission during childhood: a Danish national cohort study. Lancet Public Health 2017;2:e314-22.

50. Dooley E. Prison suicide in England and Wales, 1972-87. Br 7 Psychiatry 1990; 156:40-5.

51. Humber N, Webb R, Piper M, et al. A national case-control study of risk factors among prisoners in England and Wales [published erratum in Soc Psychiatry Psychiatr Epidemiol 2013;48:1187]. Soc Psychiatry Psychiatr Epidemiol 2013;48:1177-85

52. Australian Standard Offence Classification (Queensland Extension) (QASOC). Brisbane (Australia): Office of Economic and Statistical Research, Queensland Government; 2008. Available: www.qgso.qld.gov.au/about-statistics/statistical -standards/queensland/qasoc.php (accessed 2017 Aug. 30).
53. Landis JR, Koch GG. The measurement of observer agreement for categorical data. Biometrics 1977;33:159-74.

54. Whitlock J, Eckenrode J, Silverman D. Self-injurious behaviors in a college population. Pediatrics 2006;117:1939-48.

55. Fliege H, Kocalevent RD, Walter OB, et al. Three assessment tools for deliberate self-harm and suicide behavior: evaluation and psychopathological correlates. 7 Psychosom Res 2006;61:113-21.

56. Rockett IR, Caine ED. Self-injury is the eighth leading cause of death in the United States: it is time to pay attention. 7AMA Psychiatry 2015;72:1069-70.

57. Rockett IR, Lilly CL, Jia H, et al. Self-injury mortality in the United States in the early 21 st century: a comparison with proximally ranked diseases. 7AMA Psychiatry 2016;73:1072-81.

58. Borschmann R, Hogg J, Phillips R, et al. Measuring self-harm in adults: a systematic review. Eur Psychiatry 2012;27:176-80.

59. Thompson SC, Woods JA, Katzenellenbogen JM. The quality of Indigenous identification in administrative health data in Australia: insights from studies using data linkage. BMC Med Inform Decis Mak 2012;12:133.

60. Kariminia A, Law MG, Butler TG, et al. Factors associated with mortality in a cohort of Australian prisoners. Eur 7 Epidemiol 2007;22:417-28.

61. Spilsbury K, Rosman D, Alan J, et al. Cross-border hospital use: analysis using data linkage across four Australian states. Med 7 Aust 2015;202:582-6.

62. Rosman D, Spilsbury K, Alan J, et al. Multi-jurisdictional linkage in Australia: proving a concept. Aust NZ F Public Health 2016;40:96-7.

Affiliations: Department of Psychiatry (Borschmann), The University of Melbourne, Melbourne; Centre for Adolescent Health (Borschmann, Kinner), Murdoch Childrens Research Institute, Parkville; Melbourne School of Population and Global Health (Borschmann, Young, Snow, Kinner), The University of Melbourne, Melbourne, Australia; Department of Health Service and Population Research (Borschmann), Institute of Psychiatry, Psychology \& Neuroscience, King's College London, London, UK; Centre for Health Services Research (Young), School of Population and Global Health, The University of Western Australia, Perth; National Drug Research Institute (Young), Curtin University, Perth, Australia; Centre for Academic Mental Health (Moran), University of Bristol, Bristol, UK; Centre for Mental Health (Spittal), Melbourne School of Population and Global Health, The University of Melbourne, Melbourne; Centre for International Child Health (Snow), Department of Paediatrics, The University of Melbourne, Melbourne; The Black Dog Institute (Mok), Randwick; Mater Research Institute (Kinner), The University of Queensland, South Brisbane; Griffith Criminology Institute (Kinner), Griffith University, Brisbane; School of Public Health and Preventive Medicine (Kinner), Monash University, Melbourne, Australia; Netherlands Institute for the Study of Crime and Law Enforcement (Kinner), Amsterdam, The Netherlands

Contributors: Rohan Borschmann and Stuart Kinner conceived the study, and Stuart Kinner designed the study. Jesse Young conducted the data analyses. Rohan Borschmann drafted the manuscript, and Stuart Kinner, Jesse Young, Matthew Spittal, Paul Moran, Kathryn Snow and Katherine Mok contributed to interpreting the data and revising the manuscript accordingly. All of the authors gave final approval of the version to be published and agreed to be accountable for all aspects of the work.

Funding: The Passports study was funded through an Australian National Health and Medical Research Council (NMHRC) Strategic Award (no. 409966) and Project Grant (no. 1002463). Rohan Borschmann is supported by an NHMRC Early Career Fellowship (no. 1104644). Stuart Kinner is supported by an NHMRC Senior Research Fellowship (no. 1078168). Jesse Young is supported by a Melbourne International Research Scholarship (PhD) from the University of Melbourne.

Acknowledgements: The authors thank Queensland Corrective Services for assistance with data collection and all Passports study participants for sharing their experiences. The views expressed herein are solely those of the authors and in no way reflect the views or policies of Queensland Corrective Services.

Supplemental information: For reviewer comments and the original submission of this manuscript, please see www.cmajopen.ca/content/5/3/ E694/suppl/DC1. 